\title{
Effect of Intrinsic Foot Muscle Exercises on Foot Posture Index in Obese Individuals with Pes Planus
}

\author{
Riddhi Kate ${ }^{1}$, Anagha Palkar ${ }^{2}$ \\ ${ }^{1}$ B.P. Th, DPO's NETT College of Physiotherapy, Thane, Maharashtra, India. \\ ${ }^{2}$ Assistant Professor DPO's NETT College of Physiotherapy, Thane, Maharashtra, India. \\ Corresponding Author: Riddhi Kate
}

\begin{abstract}
Background: Flatfoot (pes planus) is a postural deformity in which the arch of the foot collapses. Obesity increases the stresses applied to the foot directly, via increased bodyweight, and indirectly, via alterations to foot structure. Extrinsic and intrinsic foot muscles act as the main components of foot function. Foot posture index is a clinical tool used to quantify the degree to which a foot is pronated, neutral or supinated. Studies done have concluded that strengthening intrinsic muscles enhances dynamic support of MLA and foot stability. There is lack of literature on effectiveness of intrinsic exercise on flat foot in obese individuals, hence the present study is undertaken to assess and evaluate the effect of intrinsic foot muscle training on Foot Posture Index in obese individuals with pes planus.

Methodology: This study was an experimental study where 30 obese patients were selected using convenient sampling.

Pre and post treatment score of foot posture index was calculated. After that intrinsic foot muscle exercises and SFE were implemented on the subjects. Protocol (2 times/day for 6weeks).

Results: This study included 30 subjects consisting of 16 females and 14 males. Intra-group comparison of right leg and left leg foot posture index revealed that the FPI score scores were significantly reduced in obese individuals at sixth week from baseline $(\mathrm{p}<0.0001)$

Conclusion: Six-week short foot exercises and intrinsic foot muscle training was effective on reducing the foot pronation, pain and there was significant reduction in the FPI score in obese individuals with pes planus.
\end{abstract}

Keywords: SFE-Short Foot Exercises, FPI- Foot Posture Index, Pes Planus, Flat foot, Obese, Intrinsic foot muscle training

\section{INTRODUCTION}

Flatfoot is also called as pes planus or fallen arches. Flatfoot i.e., acquired flatfoot develops due to injury, prolonged stress to the foot, obesity, illness, faulty biomechanics ${ }^{[1]}$. It is a postural deformity in which the arch of the foot collapses

Obesity is an issue that is becoming increasingly prominent in almost ever developed nation. Obesity affects the entire body and its effects on our feet are largely overlooked, Feet, as the body's base of support, continually endure often high ground reaction forces generated during activities of daily living. The component primarily responsible for the feet is absorbing and dissipating these forces in the longitudinal arch ${ }^{[2]}$.

Various authors also have suggested that excessive increase in weight-bearing forces caused by obesity may negatively affect the lower limbs and feet. ${ }^{[4]}$

There are a number of mechanisms by which obesity may affect the foot. These include biomechanical changes to foot structure, such as pes planus, and changes to the plantar fat pad, including increased plantar pressures, inadequate muscular 

planus.

strength and/or power, particularly in activities requiring movement against gravity, and changes in gait. ${ }^{[2]}$

It was reported that the incidence of flat foot in the population studied was found to be $16.4 \%$, of which $18.1 \%$ were males and $14.6 \%$ females. Fifty percent showed slight flat-footedness (1st degree), $28 \%$ moderate (2nd degree) and 22\% showed very marked flat - footedness (3rd degree). The frequency of obesity and overweight in the same population was found to be $27.3 \%$ $\left(\right.$ Cole index $>120^{)}$. [1]

Many factors influence the structure and functioning of the foot, one of them being body weight. Flatfoot is often a complex disorder, with diverse symptoms and varying degrees of deformity and disability ${ }^{[2] .}$

The first and perhaps most obvious effect that obesity will have on our feet is that it places a far greater amount of weight on them when we stand or move about ${ }^{[5]}$

This results not only in placing immediate physical stress on the skin and bones in our feet, but also physically alters them over time. Fallen arches are a common side-effect of obesity, additional strain on inner side of foot. This may result in a typical, functionally hyper-pronated foot

Studies show that flattening of arch causes postural instability and is also associated with musculoskeletal disorders affecting the lower limb, such as knee and hip osteoarthritis ${ }^{[3]}$

There are 10 intrinsic muscles located in the sole of the foot. They act collectively to stabilize the arches of the foot, and individually to control movement of the digits. ${ }^{[3]}$

Extrinsic and intrinsic foot muscles act as the main components of foot function and the intrinsic foot muscles are considered to have a more important role in dynamic foot control. Evidence suggests that strengthening intrinsic muscles enhances dynamic support of MLA and foot stability

Foot posture index is a clinical tool used to quantify the degree to which a foot is pronated, neutral or supinated. It is a robust measure and reliable means of static foot assessment and offers a more valid approach to assessing static foot structure ${ }^{\text {[6] }}$

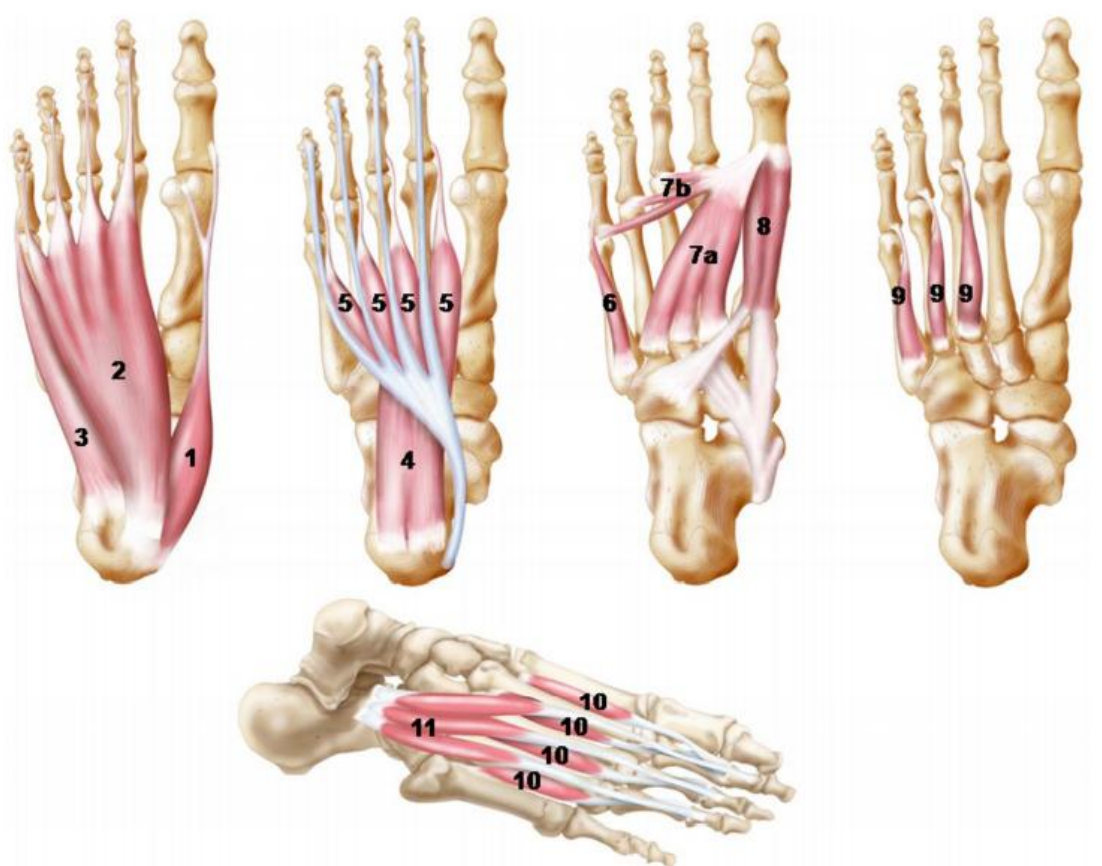

Fig 1. The intrinsic foot muscles are presented in their anatomic orientation within the four plantar layers and the dorsal intrinsic muscle. The numbers correspond to the muscles as follows: (1) abductor hallucis, (2) flexor digitorum brevis, (3) abductor digiti minimi, (4) quadratus plantae (note its insertion into the flexor digitorum tendon), (5) lumbricals (note their origin from the flexor digitorum longus tendon), (6) flexor digiti minimi, (7) adductor hallucis oblique (a) and transverse (b) heads, (8) flexor hallucis brevis, (9) plantar interossei, (10) dorsal interossei and (11) extensor digitorum brevis. [Source: McKeon PO, et al. Br J Sports Med 2014;0:1-9. doi:10.1136/bjsports-2013-092690] 

planus.

A study performed by Shree $S$, Revathi S, Thiyagarajan A, Kumar D on obese individuals which shows that intrinsic foot muscle weakness leads to pronation (pes planus) of the foot leading to painful foot conditions and balance issues.

There is a study done by Daneshmandi H, Rahnema N, Mehdizadeh $\mathrm{R}$ that suggest obesity increases the stresses applied to the foot directly, via increased bodyweight, and indirectly, via alterations to foot structure, which partly explain the link between obesity and the development of flat foot and foot pain. ${ }^{[2][1]}$

A study performed by Unver B, Erdem EU, Akbas E on individuals having flat foot that showed six-week SFE was effective on reducing ND, foot pronation, foot pain and disability in pes planus. Increase in plantar force of medial midfoot after SFE might arise from the increment in plantar loading of lateral midfoot with decreased foot pronation ${ }^{[5][8]}$

However, there is lack of literature on effectiveness of intrinsic exercise on flat foot in obese individuals, hence the present study is undertaken to assess and evaluate the effect of intrinsic foot muscle training on Foot Posture Index in obese individuals with pes planus.

\section{MATERIALS AND METHODS}

This study was an experimental study where 30 obese patients were selected using convenient sampling.

Inclusion criteria: BMI - Overweight and Obese class 1 individuals willing to participate, both males and females were included in the study between age group 35 to 50 years.

Exclusion criteria: Subjects having any recent musculoskeletal injuries or any recent trauma to the lower extremity or having any neurological problems or any recent surgeries of the lower limb.

\section{PROCEDURE:}

Obese individuals were screened and included in the study according to the inclusion criteria. The subjects were carefully explained about the procedure and a written informed consent was taken. The aim and method of the study was explained and their consent on the consent form was taken. Foot posture index was taken during the assessment. These subjects were then taught short foot exercises and intrinsic foot muscle strengthening exercises which were to be performed for 6 weeks and the pre and post foot posture values were compared.

\section{METHOD}

Foot posture index: Postural deviations were observed using foot posture index scale. In this scale talar head, supra and infra navicular angle, calcaneal curve, medial longitudinal arch, abduction /adduction of the rear foot.

For determining the foot posture index ask the subjects to stand still to observe the deviations in the angles of the foot. After taking each score on the foot posture index scale of 6 components,

$(0)$ is given for neutral, $(+2)$ is given for pronated, (-2) is supinated. The final score was a whole number between -12 to +12 .

Pre and post treatment score of foot posture index was calculated. After assessment intrinsic foot muscle exercises were implemented on the subjects. Intrinsic foot muscle exercises given as follows; Protocol (2times/day for 6weeks).

\section{Plantar fascia stretch}

- Pitting on a chair, cross the injured heel over the other leg

- hold the foot in your opposite hand

- Pull the toes toward the shin to create tension in the arch of the foot

- place the other hand on the bottom of the foot to feel for tension in the plantar fascia

- use a towel to grasp and stretch the foot if it is difficult to hold otherwise hold for 10 seconds. Repeat two to three times 


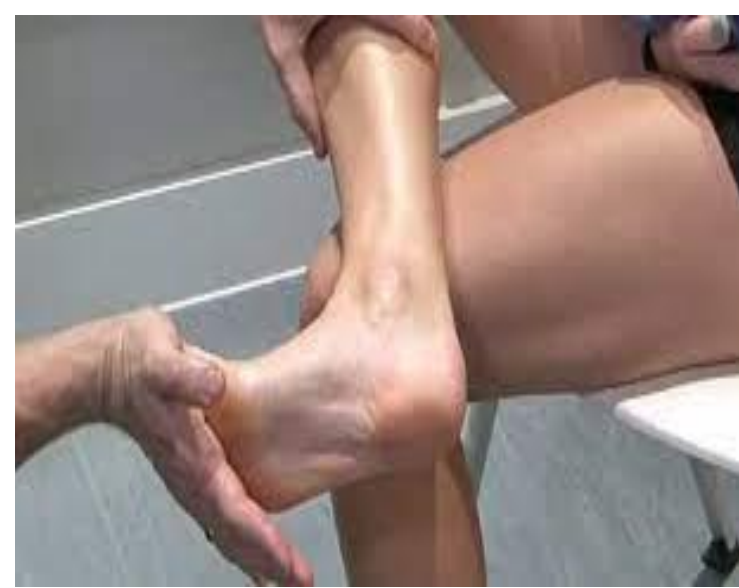

Fig.1 Plantar Fascia Stretch

\section{Short foot exercises:}

- Sit with good posture in a sturdy chair with both foot on the floor, your toes facing straight forward and your knees bent to 90 degrees.

- Ask the patient to inhale and contract the muscles on the bottom of the foot and lower legs to raise the arch of your foot without curling your toes.

- Hold this for 6 seconds and then relax. Continue this exercise for 100 repetitions in a day.
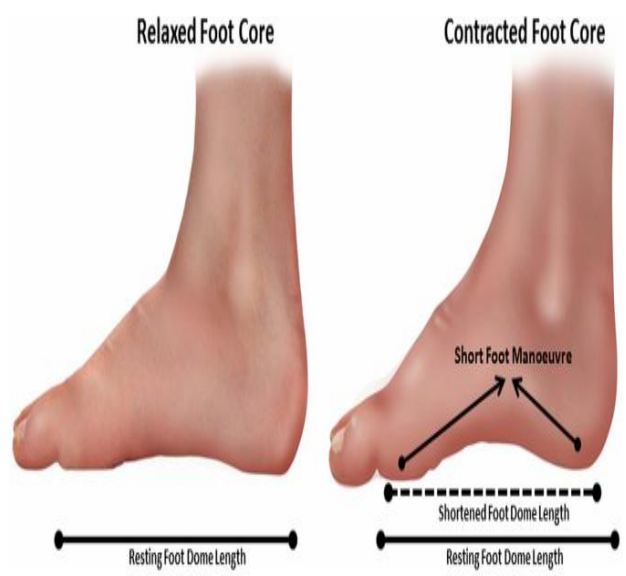

[Source: McKeon PO, et al. Br J Sports Med 2014;0:1-9. doi:10.1136/bjsports-2013-092690]

Fig.3 Short Foot Exercise

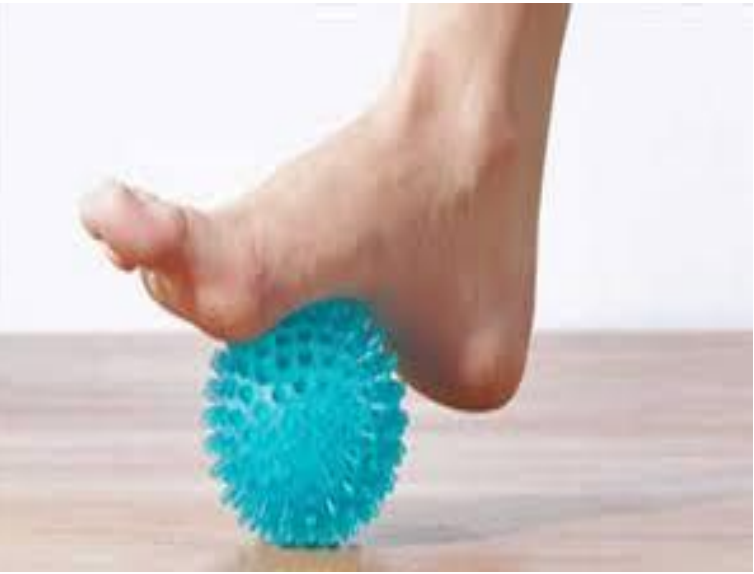

Fig. 2 Plantar Fascia Release

- Move the towel towards yourself in front of your foot. Sit with your heels under your knees.

- See to it that both the feet are parallel to each other with the toes pointing forward.

- Your heel stays in place on the towel as you reach out evenly along both sides.

- Pull the towel towards your heel, repeat the move for 100 repetitions

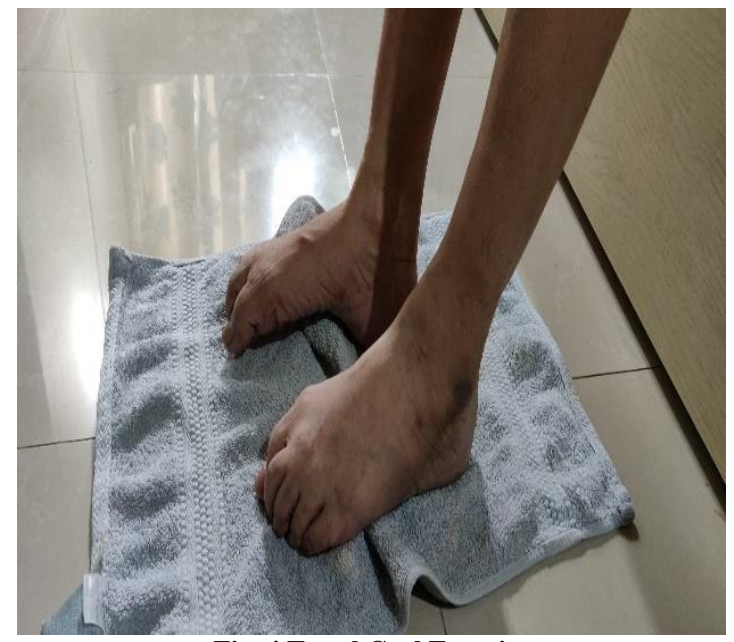

Fig. 4 Towel Curl Exercise

\section{4) Toe extension and Toe spread out exercises.}

5) Marble pickup exercise.

\section{3) Towel curl exercises:}

- Place the towel under the foot, flat. 


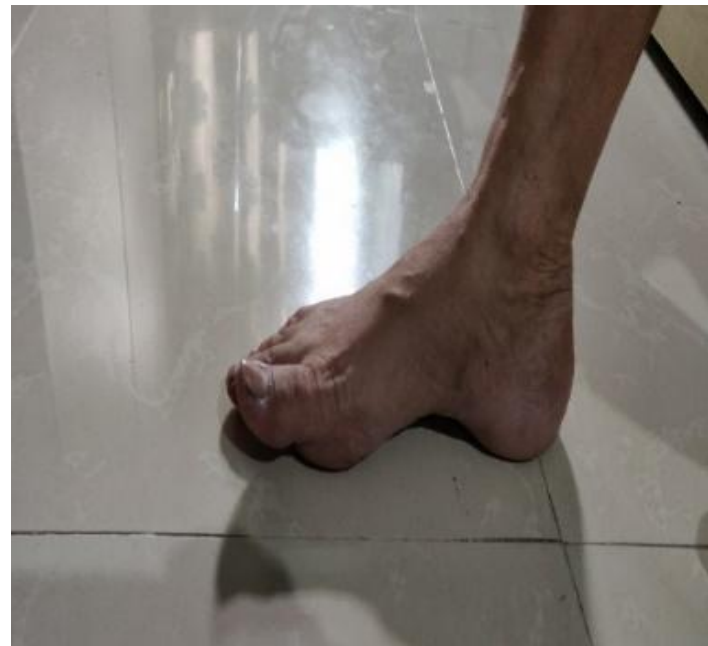

Fig. 5 Toe Extension Exercise

STATISTICAL ANALYSIS: Data was evaluated using GraphPad Prism 9.0.0. The significance level was set to $\mathrm{p}<0.05$. Normality test was conducted between the pre and the post FPI values and based on the result Non parametric test was used. Wilcoxon Signed Rank test was done to compare the pre and post FPI value where $p$ value was found to be $<0.0001$ which is significant

Table 1: Table 1. Demographic data of the participants

\begin{tabular}{|l|l|l|l|}
\hline & Mean & Std Deviation & P value \\
\hline Age & 43.00 & 4.990 & 0.0628 \\
\hline BMI & 30.32 & 2.539 & $>0.1000$ \\
\hline Gender F/M & $16 / 14$ & & \\
\hline
\end{tabular}

Interpretation: Out of the 40 samples who participated and completed the test, $55 \%$ of participants underwent isolation while $45 \%$ had been hospitalized.

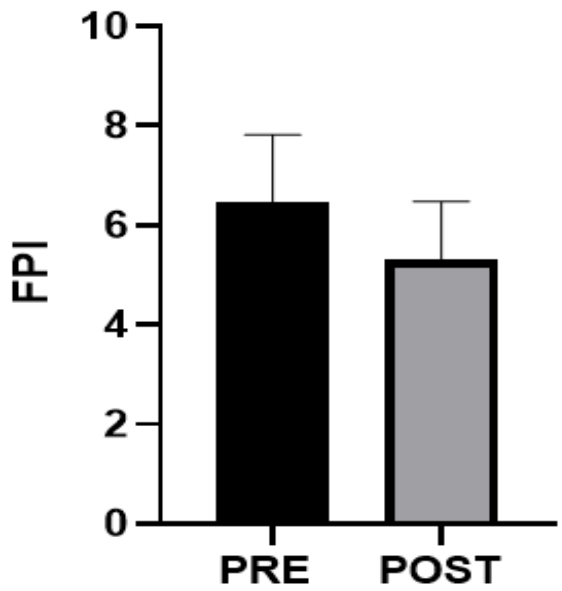

Graph 2: Left Leg: FPI values.

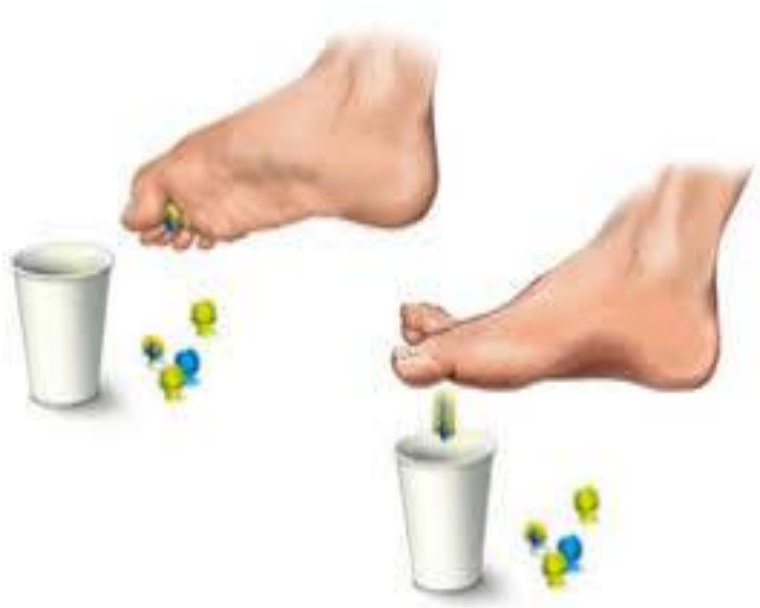

Fig. 6 Marble Pickup Exercise

Table 2 : LEFT LEG : FPI VALUES

\begin{tabular}{|l|l|l|}
\hline Variables & Pre value & Post value \\
\hline Mean & 6.467 & 5.300 \\
\hline SD & 1.358 & 1.179 \\
\hline P value & $<0.0001$ & $<0.0001$ \\
\hline
\end{tabular}

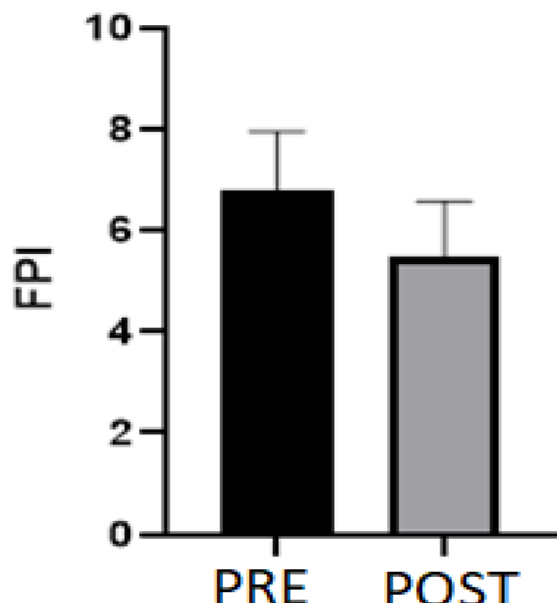

GRAPH 3: RIGHT LEG: FPI VALUES

TABLE 3: RIGHT LEG: FPI VALUES

\begin{tabular}{|l|l|l|}
\hline Variables & Pre values & Post values \\
\hline Mean & 6.800 & 5.467 \\
\hline SD & 1.157 & 1.106 \\
\hline P values & $<0.0001$ & $<0.0001$ \\
\hline
\end{tabular}

\section{RESULTS}

This study included 30 subjects consisting of 16 females and 14 males. The participants age, gender, body mass index and foot posture index Intra-group comparison of right leg and left leg foot posture index revealed that the FPI score scores were significantly reduced in obese individuals at sixth week from baseline $(\mathrm{p}<0.0001)$. 


\section{DISCUSSION}

The aim of the study is to examine the effect of intrinsic foot muscle exercises on foot posture in obese individuals with pes planus. Our findings indicate that obese adults were more likely to have a relatively pronated foot posture which was assessed using the Foot Posture Index. Obese individuals demonstrate significant changes in foot posture and range of motion, the main factor responsible for increasing loads underneath the foot was excessive bodyweight. A body of higher mass with weak musculature exerts extra forces on the arch of the foot causing weakness of the respective muscles causing collapsed arches. It significantly alters the contact surface of the arch and the ground [7]

Research revealed that intrinsic muscle activity provides support to maintenance of MLA [5] The intrinsic foot muscles are routinely active in late stance of walking and may have a significant role in controlling load distribution under the foot as well as augmenting the flexor function of the medial longitudinal arch, especially at higher speeds. Although often showing minimal activity in simple stance, the intrinsic foot muscles are more strongly recruited when additional loads are added to the participant.[8]

However local dynamic support is also thought to be provided from the intrinsic foot muscles in the active subsystems and indirectly by the contractions of the extrinsic foot muscles [8]

The local stabilizers of the foot are the plantar intrinsic muscles that both originate and insert on the foot, whereas the global movers are the extrinsic muscles that originate in the lower leg, cross the ankle and insert on the foot. While there are intrinsic muscles on both the dorsal and plantar aspects, the plantar intrinsic muscles are most commonly described due to their functional link with the longitudinal and transverse arches of the foot half dome.[8] The plantar intrinsic foot muscles consist of four layers of muscles deep to the plantar aponeurosis. The first two layers have muscle configurations which align with the medial and lateral longitudinal arches of the foot whereas the deeper layers configure more so with the anterior and posterior transverse arches [8]

Therapeutic exercise of the plantar intrinsic foot muscles has been traditionally described as occurring during toe flexion exercises such as towel curls and marble pick-ups. While these exercises certainly do activate some of the plantar intrinsic muscles, they also involve substantial activation of the flexor hallucis longus and flexor digitorum longus muscles. Recently, the 'short foot exercise' has been described as a means to isolate contraction of the plantar intrinsic muscles [8] The foot is 'shortened' by using the intrinsic muscles to pull the first metatarsophalangeal joint towards the calcaneus as the medial longitudinal arch is elevated. As the arch raises during this exercise, it is also referred to as 'foot doming'. There is increasing evidence to suggest that training the foot core via short foot exercise progressions can improve foot function [8]

There are also two studies indicating the beneficial effect of SFE on foot posture in healthy runners. [5] There are few Studies which revealed that SFE is effective on supporting the MLA in pes planus.[5] The current study exhibited that six-week SFE training had a positive impact on ND and foot posture in pes planus. Results stated that there was significant difference observed in foot posture, mainly seen as slight increase in the medial longitudinal arch height along with reduction in forefoot abduction after 6 weeks of SFE and intrinsic muscle training. Also, to some extent the talo-navicular bulging was seen on a decline. Current study also demonstrated reduction in ND and FPI after SFE. This result indicated SFE might prevent excessive pronation during gait.

Although obesity and overweight are temporary, but it can be the cause of flatfoot. Therefore, subjects should pay attention to increasing weight that may cause flatfoot and also other problems in the 
future [1]. The foot adapts itself under loading condition by maintaining the medial longitudinal arch. Increasing the loading further activates compensatory mechanisms which maintain the longitudinal arch and shifts the loads to the central and medial forefoot. [1] But treatment and prevention of flatfoot may have an effect on an individual's overall health and occurrence of other foot and ankle pathologies [1]

\section{CONCLUSION}

Our main hypothesis was supported with the current results which indicated that six-week SFE and intrinsic foot muscle training was effective on reducing, the foot pronation, pain and there was significant reduction in the FPI score in obese individuals with pes planus. Increase in plantar force of medial midfoot after SFE might arise from the increment in plantar loading of lateral midfoot with decreased foot pronation.

The present study has some limitations, Gender ratio of the groups was different; and, this might affect the outcomes.

However, the baseline outcomes of the groups were similar. This study doesn't take into account the plantar pressures of the foot in obese individuals with pes planus and thus further studies can be done on the same.

Studies can also be done on examining the effect of intrinsic muscle exercises and SFE on the plantar pressures in obese individuals with pes planus. Group assignments were conducted based on the willingness of the participants in order to increase the adherence to the exercises.

Besides, long term efficacy of intrinsic foot muscles training was not evaluated in this study. Finally, more accurate results about the effects of intrinsic foot muscle training and SFE on FPI, could be obtained if a larger number of participants were included.

In the study we found that long term intrinsic muscles strengthening and short foot exercises have proven to have a good impact over the foot posture of the obese people with pes planus,

Pes planus in obese patients leads to secondary impairments like impaired balance, pain in lower extremities compensatory changes in the lower extremities leading to functional impairments and also in patients with knee Osteoarthritis where it worsens the knee pain and aggravates the condition.

Thus, in clinical practice; along with the conventional methods of treatment for pain relief, and balance training and strength training in patients with degenerative lower extremity conditions, focus can also be given on their foot posture and can be corrected and improved by performing these short foot exercises and Intrinsic muscle training on a long term.

\section{Acknowledgement: None}

\section{Conflict of Interest: None}

\section{Source of Funding: None}

\section{Ethical Approval: Approved}

\section{REFERENCES}

1. Chougala A, Phanse V, Khanna E, Panda S. Screening of body mass index and functional flatfoot in adult: an observational study. Int J Physiother Res. 2015;3(3):103741.

2. Shree S, Revathi S, Thiyagarajan A, Kumar D. Does Obesity Cause Flat Foot?. J Obes Ther 2: 1. of. 2018;4:2.

3. Andhare N, Yeole U, Tannu MM. Effect of Intrinsic Muscle Training on Balance in Bharatanatyam Dancers: Randomized Control Trial. International Journal of Science and Healthcare Research (IJSHR) Vol.3; Issue: 4; Oct.-Dec. 2018

4. Daneshmandi H, Rahnema N, Mehdizadeh R. Relationship between obesity and flatfoot in high-school boys and girls. International Journal of Sports Science and Engineering. 2009;3(1):43-9.

5. Unver B, Erdem EU, Akbas E. Effects of short-foot exercises on foot posture, pain, disability, and plantar pressure in Pes 

planus.

Planus. Journal of sport rehabilitation. 2019 Oct 18;29(4):436-40.

6. Redmond AC, Crosbie J, Ouvrier RA. Development and validation of a novel rating system for scoring standing foot posture: the Foot Posture Index. Clinical biomechanics. 2006 Jan 1;21(1):89-98.

7. Butterworth PA, Urquhart DM, Landorf KB, Wluka AE, Cicuttini FM, Menz HB. Foot posture, range of motion and plantar pressure characteristics in obese and nonobese individuals. Gait \& posture. $2015 \mathrm{Feb}$ 1;41(2):465-9.

8. McKeon PO, Hertel J, Bramble D, Davis I. The foot core system: a new paradigm for understanding intrinsic foot muscle function. British journal of sports medicine. 2015 Mar 1;49(5):290-.

9. Okamura K, Fukuda K, Oki S, Ono T, Tanaka S, Kanai S. Effects of plantar intrinsic foot muscle strengthening exercise on static and dynamic foot kinematics: A pilot randomized controlled single-blind trial in individuals with pes planus. Gait \& posture. 2020 Jan 1;75:40-5.

How to cite this article: Kate R, Palkar A. Effect of intrinsic foot muscle exercises on foot posture index in obese individuals with pes planus. Int $J$ Health Sci Res. 2021; 11(10): 280-287. DOI: https://doi.org/10.52403/ijhsr.20211037 